\title{
Social Trust in a Familiar Community
}

\author{
Eseosa Oshodin, Francisco Chiclana and Samad Ahmadi
}

\begin{abstract}
Most computation of social trust have been used for maintaining communities of individuals, based on their past activities. The behaviour of idle individuals or non-contributors to the communities have been totally ignored in the computation as they might affect the representation of the trust computed for the individuals. If the trust for an individual have been misrepresented, other individuals in the community will erroneously disengage or engage with the individual. In this paper, a new trust metric is proposed which is based on user's pattern of interaction that will be able to assist other users in their decision making on whether to join or leave a community. Different trust features are analysed to evaluate trust values for each user, which is used to determine the trust communities of the users.
\end{abstract}

\section{Introduction}

In the context of social network, people tend to have similar preferences with their trustworthy friends and also disclose their personal information to them. According to Nepal [7], trust between people can be described as a "social element" for engagement, acceptance and other decisions. Trust is important to everyone's daily life-activities. For example, before a person accepts the help of another person/group or a group decides to accept a person, they will require to know the trustworthiness of that person or group. This trustworthiness of people form familiar communities where their relationship can maintained and it can also be referred to for trust information, which can assist in decision making. Examples of the familiar communities exist in Facebook and twitter, where users become familiar after several interactions or engagements with themselves. But when trust information is unknown to a person, an inaccurate decision or no decision may be made by the person.

Eseosa Oshodin, Francisco Chiclana and Samad Ahmadi

De Montfort University, Leicester, e-mail: eseosa.oshodin, chiclana, sahmadi@dmu.ac.uk 
Sociologists, such as Luhmann [5], referred to trust as a means for building familiar communities to reduce complexity when different individuals have diverse views, opinions and goals. But Psychologists tend to define trust as the belief for a person to behave as expected. According to Erikson [2], trust emerges from the first stage of human life (0-2 years) where infants learn how to depend and rely on other people to satisfy their needs but when the infants are neglected by other people, they will learn the mistrust as they view this people to be undependable and unreliable. Both experience and familiarity of the infants with other people will enable them to discern similar situations in the future.

In general, trust is a conditional means to portray individuals' behaviour based on their previous pattern of interaction. In other words, it is the probability that future behaviour of individuals will be faultless and similar to their previous true behaviour in all situations. From this definition, three factors can be drawn out: familiarity, experience and honesty. Familiarity factor reduces the complexity of the dynamic nature of the community [5], the experience factor reflect the similarity in behavioural patterns, while the honesty factor controls the representation of the behavioural patterns in a familiar community. A new trust model will be introduced which relies on these factors.

The rest of the paper is structured as follows: Section 2 provides a review on the relevance of trust in communities and the relationship between familiarity, experience and trust. Methods for evaluating familiarity and experience from previous research are also discussed to compare their various features. Section 3 introduces the proposed approach for trust computation that will assist in observing users behaviour. Finally, Section 4 summarizes this study with its discoveries.

\section{Literature Review}

\subsection{Trust, Experience and Familiarity}

The definition of trust by Rutter [10] (cited in [1]) referred to trust as a "generalized expectancy" from an individual or group given to another individual or group to fulfil tasks. In other words, trust from agent $A$ to agent $B$ is the belief in the ability of agent $B$ to accomplish given tasks to an expected and satisfactory level. However, users may still remain in a state of uncertainty based on their engagements with others.

Hardin [3] described trust between nodes in his game experiment to be asymmetric and referred to it as a one-way trust (see Fig.1). This type of trust exists when either of the nodes (initiator) decides to act first based on its trust towards the other node (respondent) and the respondent might decide to either respond to or ignore the action of the initiator based on its own trust towards the initiator.

A different view of trust by Marsh [6] states that trust is a useful tool for discernment of the behaviour of other individuals based on previous experience. In [10], it 
was claimed that trust can be based on "local experience" (experience from engagement between two individuals within a community), interaction and daily routine of individuals which can further improve the participation of others. From these definitions, trust based on experience can be used as a criteria to consider a user into an active community. That is, without its existence, communities will cease to evolve or might completely disappear.

Marsh [6] also stated that in order to determine the outcomes from new diverse situations of interactions, we will need to observe the behaviour of existing ones which were affected by their "importance". He described this "importance" as a measure of the expected benefits to be gained from situations. This importance factor is believed by Marsh [6] to cause an increase in trust based on the fact that as a situation becomes important, the choice for cooperation amongst individuals increases. The importance of individuals should also be considered to determine if the individuals will be able to fulfil the tasks in future situations.

Wu \& Chiclana [13] determined the importance degree of an individual by considering the trust degree and consensus level of the individual in a social network. The importance degree was used in evaluating the weights for each individual in order to aggregate the individual preference relation into a collective one. This collective preference relation is then used for giving suggestions or advices to individuals in the same group. If a user doesn't know the preference of another individual who has no or few engagements in the past, the user can use the collective mechanism to decide whether to trust this individual.

According to Luhmann [5], trust can also exist in a familiar community and there may be changes in the behaviour of members in that community. If these changes occur, the existing members might have to either adapt or disengage from the community. External non-members may decide to join the community based on either their high interest in the new characteristics of the community or their familiarity with the exemplar of that community. High interest in sincere characteristics of the community motivates the non-members to initiate communication with members of that community based on their belief in the importance of the community to meet their needs.

In the communication field, Gerck [16] defined trust as an important tool for a communication path but it cannot be transferred from a source to destination through the same path. This means that an individual will rely on information through a channel or path from another individual (source) based on trust inferred from multiple paths relating the source with other individuals in a community. In other words, trust of agent $A$ for agent $B$ can be seen as a rationale for accepting information from

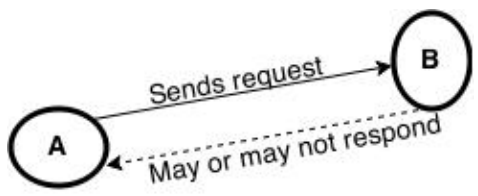

Fig. 1 One-way trust network 
agent $B$ based on agent $B$ 's previous experiences with others in a community. Gerck [16] still went further to refer trust as a reasonable "collective agreement" which means that there should be equivalent-honest patterns which can be aggregated to make accurate judgement.

\subsection{Evaluation of Familiarity and experience}

Previous research work in [15] used both Familiarity and Experience as parameters for modelling trust. The familiarity value was initially measured based on the work by Carter \& Ghorbani [1], where similarity values were also considered in the measurement, since two individuals can become friends when they have similar characteristics or behaviour. The similarities between agents were determined using equation (1) which is based on Hamming distance of value hierarchies. This hierarchy values represent the importance value from each agent given to other agents in a community. An example of the similarity evaluation will be shown later in the proposed model of this current paper.

$$
S_{\left(a, a_{i}\right)}=1-\frac{H_{\left(\tau_{a}, \tau_{a_{i}}\right)}}{N}
$$

Where: $\tau_{a}$ and $\tau_{a_{i}}$ are hierarchy values for agent $\mathbf{a}$ and agent $\mathbf{a}_{i} ; H_{\left(\tau_{a}, \tau_{a_{i}}\right)}$ is the total number of distinction between the hierarchy values for agent $\mathbf{a}$ and $\mathbf{a}_{i} ; N=$ Total number of hierarchy values given to the agents.

These similarity values were used in [15] to initialize familiarity when an individual (for example, a customer) $\mathbf{u}$ has not had any encounter before with another individual (selling agent) a. Thus, the initial familiarity $F_{o(u, a)}$ was then evaluated by considering other previous engagements the individual $\mathbf{u}$ had with other individuals (selling agents) $\mathbf{a}_{i}$, using weighted average functions (as shown in equation (2)) [15].

$$
F_{o(u, a)}=\sum_{i=1}^{n}\left[F_{\left(u, a_{i}\right)} \cdot \frac{S_{\left(a, a_{i}\right)}^{2}}{\sqrt{\sum_{i=1}^{n} S_{\left(a, a_{i}\right)}^{2}}}\right]
$$

Where: $F_{\left(u, a_{i}\right)}$ is the existing familiarity value for user $\mathbf{u}$ with other agents $\mathbf{a}_{i}$; $S_{\left(a, a_{i}\right)}$ is the similarity value between the target agent $\mathbf{a}$ with other agents $\mathbf{a}_{i}$.

It is possible to evaluate $F_{o(u, a)}$ using previous engagement feedbacks, as we need to predict the familiarity value for each users since their behaviours in a social community is dynamic in nature.

Previous research [7] referred to "popularity trust" as a value given to a member of a community by other members of that same community. This can represent the familiarity value for the member since it is based on existing association with other members. This value was evaluated with equation (3) which is based on the positive and negative feedbacks given to the member by other members of the community, after this member had previously carried out an active or passive interactions to- 
wards these other members. For instance, if a user sends an email towards another user, it can be referred to as an active interaction; while if a user views another user's profile page, it can be referred to as a passive interaction. The positive feedbacks for an active interaction will then be the response by replying to the email previously sent, while that for a passive interaction will be the response by viewing on the other individual's page. The negative feedbacks is the number of non-response to previous emails or viewing actions.

$$
F_{(a) p o p}^{x}=\frac{\sum_{b=1}^{|M|-1} \frac{\left|I_{a b}^{x+}\right|+1}{\left|I_{a b}^{x+}\right|+\left|I_{a b}^{x-}\right|+2}}{|M|-1}
$$

Where: $F_{(a) \text { pop }}^{x}$ is the popularity value given to user $\mathbf{a} \in \boldsymbol{U}$ on a situation $\mathbf{x} ;|M|$ is the number of members in the community $\boldsymbol{U} ;\left|I_{a b}^{x+}\right|$ and $\left|I_{a b}^{x-}\right|$ represent the number of positive feedbacks and negative feedbacks respectively, given by user $\boldsymbol{b} \in \boldsymbol{U}$ on user a's interactions on situation $\mathbf{x}$.

This formalization of popularity value was based on the research by Josang \& Ismail [4] where beta probability distribution function was used since a choice (either positive or negative) on a situation or context $\mathbf{x}$ is uncertain. This evaluation is related to Laplace rule of succession [14] where both success and failure outcomes are considered. This popularity value relies on the Probability expectation value [4], $\operatorname{Prob}_{(E)}$, which is evaluated using two parameters, $\alpha$ and $\beta$ (as shown in equation (4)), related to the number of positive feedback and negative feedback received, respectively.

$$
\operatorname{Prob}_{(E)}=\frac{\alpha}{\alpha+\beta}
$$

From equation (3),

$$
\begin{aligned}
& \alpha=\left|I_{a b}^{x+}\right|+1 \\
& \beta=\left|I_{a b}^{x-}\right|+1
\end{aligned}
$$

Josang [4] referred to $\left|I_{a b}^{x+}\right|$ and $\left|I_{a b}^{x-}\right|$ as the degree of satisfaction and the degree of dissatisfaction respectively, whereby $\left|I_{a b}^{x+}\right|$ measures the level of success in user a's actions related to situation $\mathbf{x}$ while $\left|I_{a b}^{x-}\right|$ measures the level of failure in user a's actions related to situation $\mathbf{x}$.

Substituting $\alpha$ and $\beta$ into equation (4), the probability of the positive feedback to be true in the future, $\operatorname{Prob}_{\left(E_{a b}^{+}\right)}$will then be:

$$
\operatorname{Prob}_{\left(E_{a b}^{+}\right)}=\frac{\left|I_{a b}^{x+}\right|+1}{\left|I_{a b}^{x+}\right|+\left|I_{a b}^{x-}\right|+2}
$$

From equation (3), the popularity value for each individual can then be evaluated as:

$$
F_{(a) p o p}^{x}=\frac{\sum_{b=1}^{|M|-1} \operatorname{Prob}_{\left(E_{a b}^{+}\right)}}{|M|-1}
$$


This estimated-popularity value using equation (3) reveals how well that user is known to the community based on the existing interactions. If users have few interactions with the community members, their familiarity value could be predicted based on their similar members' previous interactions with other members.

Nepal [7] also went further to evaluate engagement value based on both active and passive engagement interactions, whereby the number of responses to a user's message is consider. The engagement value $E_{(a) C}^{x}$ was also evaluated using the beta probability function (as shown below) which is similar to equation (3) since it also depends on the positive feedbacks and negative feedbacks for its evaluation.

$$
E_{(a) C}^{x}=\frac{\sum_{b=1}^{|M|-1} \frac{\left|C_{a b}^{x+}\right|+1}{\left|C_{a b}^{x+}\right|+\left|C_{a b}^{x-}\right|+2}}{|M|-1}
$$

Where: $\left|C_{a b}^{x+}\right|$ and $\left|C_{a b}^{x-}\right|$ represent the number of positive and negative feedback respectively, given by user $\mathbf{a}$ on user $\boldsymbol{b}$ 's interaction on situation $\mathbf{x} ;|M|$ is the number of members in the community.

This evaluation depends more on the degree of satisfaction because the degree of satisfaction increases the experience level of a user which obeys the theory in [11] that reveals the performance from an event or situation affecting the experience level. The experience level measures how much a user of a community has satisfied other users of the same community after several interactions. If a user a sends number of posts or messages to another user $\mathbf{b}$, the number of views (passive interactions) or replies (active interactions) to those messages can be considered as the degree of satisfaction for user $\mathbf{b}$ who viewed or responded to the messages. This degree value is given to user $\mathbf{b}$ by user $\mathbf{a}$ who evaluates the positive feedbacks received on its messages. Whereas, the number of messages that were not viewed or had no response can be considered as the degree of dissatisfaction $\left(C_{a b}^{x-}\right.$ from equation (9)).

From both evaluation of Familiarity and experience with equation (3) and equation (9) respectively, the sincerity of the individuals was not considered, as the truthfulness of their feedbacks were not evaluated.

\section{Modelling Trust from a Community}

In this section, the proposed trust metric is presented and tested with the data provided in the Appendix. If a new user joins a community of other users or has had few interactions with other users in the community, the social trust information is required to assist in deciding on engagement with the user. 


\subsection{Familiarity-Experience Based Social Trust}

Familiarity between individuals will be established when one of the individual believes that the other individual is important in fulfilling a task. Since the familiarity between two individuals can be uncertain, the similarity can reveal the probability of this two individuals to be familiar with each other in the future $[1,15]$. The similarity in behaviour can be used in the comparison between a particular individual and another member in a community, by viewing the way they both consider the importance of other members of the community in fulfilling various tasks. Hence, the familiarity between two individuals can be represented with their similarity value.

As earlier discussed, the similarity values between individuals can be evaluated using equation (1), where the difference in hierarchy of importance value for each member is considered. For example, given a hierarchy table which can be used to evaluate the similarity between node $\mathbf{V}_{1}$ and $\mathbf{V}_{3}$ as shown in table $\mathbf{1}$, where the evaluation of the importance value given to each individual by node $V_{1}$ and $V_{3}$ will be discussed later in this paper.

Table 1 Hierarchy table with Importance value for each node

\begin{tabular}{|c|cccc|}
\hline Node & $V_{1}$ & $V_{3}$ & $V_{5}$ & $V_{32}$ \\
\hline$V_{1}$ & 0 & -3 & 0 & 0 \\
$V_{3}$ & 3 & 1 & 0 & 11 \\
\hline
\end{tabular}

Using equation (1) on table 1's data, the similarity value between $V_{1}$ and $V_{3}$ is:

$S\left(V_{1}, V_{3}\right)=1-\frac{H_{\left(\tau_{V_{1}}, \tau_{V_{2}}\right)}}{N}=1-\frac{3}{4}=0.25$

The familiarity value for an individual $\mathbf{F}_{i}$ is therefore evaluated as the mean of similarity values $\mathbf{S}_{i j}$ between the individual and other members of the community (as shown in equation (10)) as it reveals the central value of the probability distribution for familiarity. This value denotes the possibility of the individual to be contacted in the future by members of the community. It ranges between 0 and 1 , where the value 0 denotes that there is the possibility that an individual will have no or few interaction with other individuals in the future while the value 1 denotes that the individual will definitely be familiar with other members in the network.

$$
F_{i}=\frac{\sum_{i=1}^{j=n} S_{i j}}{n}
$$

Where $n=$ the number of members $\boldsymbol{j}$ from the community that have interacted with the individual $\mathbf{i}$

The experience value for a user can be defined as the likelihood of the user to understand the behaviour of other members in a community. If user $\mathbf{i}$ has a task to fulfil for another user $\mathbf{j}$, the outcome from the roles might either be successful or unsuccessful to a certain degree. The experience value for user $\mathbf{i}$ can be evaluated using equation (9), whereby all user i's engagement outcomes (feedbacks) with other members of the community are considered in discerning their behavioural pattern. 
The overall engagement outcome is then referred to as user i's experience value for the whole community with values ranging $[0,1]$, where the value 0 denotes that the user in the community will have impartial views on other members' behaviour while the value 1 denotes that the user will definitely understand other members' behaviour in the community.

As earlier discussed, both familiarity and experience contribute to trust computation, as they exist within a probabilistic metric space ${ }^{1}$. Thus, the trust value of an individual $S T_{i}$ can be defined as the output from both familiarity value and experience value inputs. As both inputs are in the range [0,1], the output can be modelled with a conjunction operator on function domain $[0,1] \times[0,1]$, which can be generalized using a t-norm operator ${ }^{2}$. A T-norm type, product t-norm $T_{\text {prod }}$ is chosen here for the trust evaluation, as it is differentiable so it assures smooth transitions in changes on any of the input values. $\mathrm{E}$,

Using T-norm function $T:[0,1] \times[0,1] \rightarrow[0,1]$ as a product T-norm for all $\mathrm{F}$ and

$$
T_{\text {prod }}(F, E)=F \cdot E
$$

then

$$
\begin{gathered}
S T_{i}=F_{i} \cdot E_{i} \\
S T_{i}=\frac{1}{n^{2}} \sum_{i=1}^{j=n} F_{i j} \cdot E_{i j}
\end{gathered}
$$

Where: $F_{i j}$ and $E_{i j}$ are the respective familiarity and experience between user $i$ and $j ; F_{i}$ and $E_{i}$ are the overall familiarity and experience values respectively; $n$ is the number of interaction between user $i$ and $j$.

Another factor which can be consider in the trust computation is the honesty value $H_{i}$ which is given to an individual by the community. According to [1], honesty factor encourages trust between pairs of individuals, as it is believed that a honest individual will not defame or misrepresent the character of another individual. We can then define honesty factor as the degree of representation to an item's characteristic or preference. For instance, if user $\mathbf{i}$ sends 5 messages to user $\mathbf{j}$ but user $\mathbf{j}$ only responded to or accepted 4 of the messages, then it is believed that 0.2 of the sent messages denote that user $\mathbf{j}$ must have misrepresented the preference or characteristic of user $i$. The overall honesty value for an individual (as shown in table 5) can be determined by considering the number of times different users' preference is misrepresented by the individual based on various patterns of their engagements.

Therefore, an improved social trust value for an individual $\alpha S T_{i}$ which requires the honesty factor can be defined as the dot product of the individual's familiarity, experience and honesty values (as shown below). Both $S T_{i}$ and $\alpha S T_{i}$ are in the range $[0,1]$ with 0 denoting a no-trust status for the individual while 1 denotes an absolute

\footnotetext{
${ }^{1}$ In mathematics field, Probabilistic metric space is the generalization of metric spaces whereby the distances between points are defined by probability distribution.

${ }^{2}$ T-norm is a binary operation that can be used in generalizing the conjunction within probabilistic metric spaces and it satisfies the commutative, monotonic, associative and identity (where the neutral element is 1) properties.
} 
Social Trust in a Familiar Community

trust status for the individual.

$$
\alpha S T_{i}=F_{i} \cdot E_{i} \cdot H_{i}=S T_{i} \cdot H_{i}
$$

\subsection{Collection of Data}

From the original dataset in [8], 44 samples of interactions between 15 users (as shown in table 6 in the Appendix) were randomly selected to carry out experiments to observe the behaviour of the users. The interaction between members of the network is the exchange of messages amongst themselves, where the number of messages sent $\left(\mathbf{V}_{3}\right)$ by a member $\left(\mathbf{V}_{1}\right)$ to another member $\left(\mathbf{V}_{2}\right)$ occurred at different times. $V_{2}$ 's number of responses $\left(\mathbf{V}_{4}\right)$ to the number of messages previously sent $\left(V_{3}\right)$ by member $V_{1}$ can be assumed to be the number of messages from $V_{2}$ to $V_{1}$ represented in other situations (as shown in table $\mathbf{2}$ and table $\mathbf{3}$ ), where the initial $V_{1}$ (initial sender of messages) becomes $V_{2}$ while the initial $V_{2}$ (initial receiver of messages) then becomes $V_{1}$.

Table 2 Node 1's Interaction with other nodes

\begin{tabular}{|l|l|l|l|}
\hline $\mathbf{V}_{\mathbf{1}}$ & $\mathbf{V}_{\mathbf{2}}$ & $\mathbf{V}_{\mathbf{3}}$ & $\mathbf{V}_{\mathbf{4}}$ \\
\hline 1 & & 2 & 1
\end{tabular}

\begin{tabular}{|l|l|l|l|}
\hline 1 & 2 & 1 & 0 \\
\hline 1 & 3 & 32 & 35 \\
\hline
\end{tabular}

\begin{tabular}{lllll}
1 & 3 & 32 & 35 \\
\hline
\end{tabular}

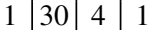

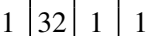

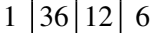

\begin{tabular}{l|l|l|l|}
1 & 44 & 5 & 4 \\
\hline
\end{tabular}

For instance, from a directed network shown in Fig.2 (extracted from table 6), a situation when node $\mathbf{1}$ sends $\mathbf{3 2}$ messages to node $\mathbf{3}$ denotes the interaction where

Fig. 2 Directed network for node 1's Interactions

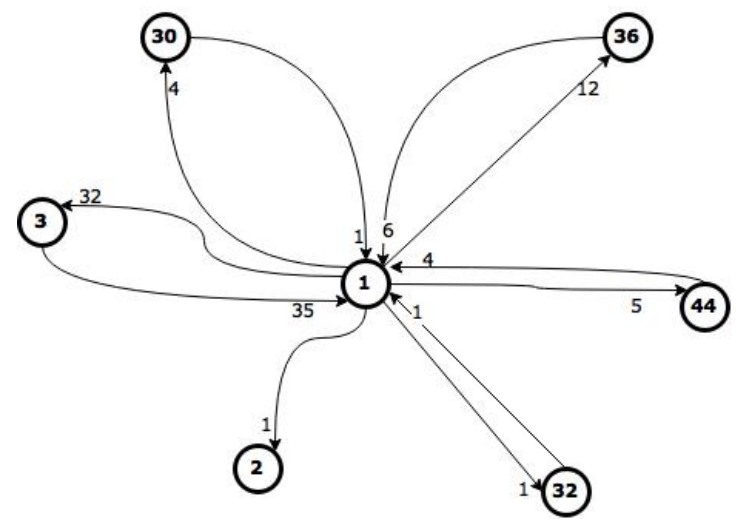


node 1's 32 messages were sent at different times to node 3 while the situation when node $\mathbf{3}$ sends $\mathbf{3 5}$ messages to node $\mathbf{1}$ denotes the interaction where node 3 provided 35 responses to node 1's messages at different times. These situations can also be viewed in the reverse way where node 3 is initially considered as the sender of the messages and the node 1 is seen as the receiver of the messages. These information on both the number of sent messages and the number of responses to the messages are then represented in table 2 and table 3. Other individual nodes' interaction data in the network were also obtained in similar pattern as table 2 and table 3.

Table 3 Node 3's Interaction with other nodes

\begin{tabular}{|c|c|c|c|}
\hline $\mathbf{V}_{\mathbf{1}}$ & $\mathbf{V}_{\mathbf{2}}$ & $\mathbf{V}_{\mathbf{3}}$ & $\mathbf{V}_{\mathbf{4}}$ \\
\hline 3 & 1 & 35 & 32 \\
3 & 2 & 7 & 0 \\
3 & 9 & 1 & 0 \\
3 & 26 & 4 & 0 \\
3 & 32 & 19 & 8 \\
3 & 36 & 9 & 5 \\
3 & 44 & 5 & 1 \\
\hline
\end{tabular}

\subsection{Analysis with Data}

With this data given in table 2 and table 3 along with other nodes' similar interaction data, both the Familiarity and Experience values for each node were further evaluated using equation (10) and equation (9) respectively. As earlier discussed, the similarities between pairs of individuals in the network required the importance values for the evaluation of each individual's familiarity value. The importance value $I V_{(i j)}$ for node $\mathbf{i}$ given by node $\mathbf{j}$ was determined by calculating the difference between the number of messages sent by node $j$ and the number of messages received by node $i$.

$$
I V_{(i j)}=m s_{(i j)}-r e s_{(j i)}
$$

Where: $m s_{(i j)}$ is the number of messages sent by node $j$ to node $i$ while res $(j i)$ is the number of messages from node $j$ received by node $i$.

The importance value for each individual in the network is then represented in a matrix where the assigners of importance value are the row variables of the matrix while the receivers of importance value are the column variables of the matrix. The number of distinction between the importance values $\left(H_{\left(\tau_{i}, \tau_{j}\right)}\right)$ given by $i$ and $j$ which is required for the evaluation of similarity values using equation (1) can be determined from this matrix. Therefore, using equation (10), the familiarity values for each individual is evaluated. 
Also, with the generated data (table 2, table 3 and similar relational tables for other nodes' interaction), the honesty values for each nodes were also evaluated (equation (16)) as the ratio of the total number of cases with response count (number of responses) being less than number of messages sent, $C N_{\text {res }<m s}$ to the total number of interaction $N_{\text {int }}$, in respect to a particular node's (node $i$ ) interaction with other members (node $j$ ) in a network.

$$
H_{i}=\left(\frac{C N_{r e s<m s}^{i j}}{N_{i n t}^{i j}}\right)
$$

The social trust value for each individual were evaluated with and without the honesty values using equation (12) and equation (14) respectively for each individual in the network. The results obtained from these evaluations, generated using the $\mathrm{R}$ software environment [9] ${ }^{3}$ were all represented in separate tables as shown in tables 4 and 5.

Table 4 Trust without Honesty factor

\begin{tabular}{|c|c|c|c|}
\hline Node & $\mathbf{F}$ & $\mathbf{E}$ & $\mathbf{T}$ \\
\hline 1 & 0.5238 & 0.5746 & 0.3009 \\
2 & 0.6000 & 0.0000 & 0.0000 \\
3 & 0.4238 & 0.3947 & 0.1673 \\
5 & 0.6857 & 0.3333 & 0.2285 \\
6 & 0.6571 & 0.5000 & 0.3285 \\
7 & 0.5762 & 0.0000 & 0.0000 \\
8 & 0.5476 & 0.5347 & 0.2928 \\
9 & 0.6143 & 0.1899 & 0.1166 \\
19 & 0.6143 & 0.3333 & 0.2047 \\
26 & 0.6286 & 0.3333 & 0.2095 \\
30 & 0.6905 & 0.5555 & 0.3836 \\
32 & 0.4809 & 0.5666 & 0.2725 \\
36 & 0.4381 & 0.3799 & 0.1664 \\
44 & 0.5000 & 0.4147 & 0.2073 \\
90 & 0.6667 & 0.6660 & 0.4440 \\
\hline
\end{tabular}

From Fig.3, the boxplot [12] compares the trust without honesty values and the trust with honesty values. This shows that the honest trust data (table 5) has the property of a Platykurtic distribution [12], as it has lower excess kurtosis ${ }^{4}$ which is in contrast to the other boxplot for non-honest trust data (table 4) that has a normal distribution property. This denotes that the Honest trust data has higher variability in the values than the non-honest trust data, as they are lesser around the mean value. This means that the data with honesty values will enable users to accurately distinguish from any other user they intend to engage with. From table 5, a node with

\footnotetext{
${ }^{3}$ Personalized functions can be created in R environment to operate on data for obtaining accurate results which can be used in further analysis.

${ }^{4}$ A lower excess kurtosis is the peakedness measurement with negative value (lower peak) that is less than zero kurtosis value for a normal distribution.
} 
Table 5 Trust with Honesty factor

\begin{tabular}{|c|c|c|c|c|}
\hline Node & $\mathbf{F}$ & $\mathbf{E}$ & $\mathbf{H}$ & $\alpha \mathbf{T}$ \\
\hline 1 & 0.5238 & 0.5746 & 0.6667 & 0.2006 \\
2 & 0.6000 & 0.0000 & 0.0000 & 0.0000 \\
3 & 0.4238 & 0.3947 & 1.0000 & 0.1673 \\
5 & 0.6857 & 0.3333 & 1.0000 & 0.2285 \\
6 & 0.6571 & 0.5000 & 0.5000 & 0.1643 \\
7 & 0.5761 & 0.0000 & 0.0000 & 0.0000 \\
8 & 0.5476 & 0.5347 & 0.2500 & 0.0732 \\
9 & 0.6142 & 0.1899 & 1.0000 & 0.1166 \\
19 & 0.6142 & 0.3333 & 1.0000 & 0.2047 \\
26 & 0.6285 & 0.3333 & 1.0000 & 0.2095 \\
30 & 0.6904 & 0.5550 & 0.0000 & 0.0000 \\
32 & 0.4809 & 0.5666 & 0.4000 & 0.1089 \\
36 & 0.4381 & 0.3799 & 0.7142 & 0.1188 \\
44 & 0.4999 & 0.4147 & 0.5000 & 0.1037 \\
90 & 0.6667 & 0.6660 & 0.0000 & 0.0000 \\
\hline
\end{tabular}

definite honest $(H=0)$ values for its familiarity and experience will have an impartial trust values (i.e $\alpha S T=0$ ). This situation denote that both the familiarity value of the node given by the members of the network and the experience or inexperience value of the node given to the whole members of the network were accurately represented and assigned to a certain degree, as it can be seen with node 2,7,30 and 90 of the network. This also shows that as these nodes are definitely honest, they will be considered by other nodes for future engagements, irrespective of their trust degree.

Other nodes with certain degrees of dishonesty for both familiarity and experience values also acquired certain degree of trust. The nodes with definite dishonest values will have higher trust values which signifies that these nodes were crafty in their behaviours to earn trust from others.

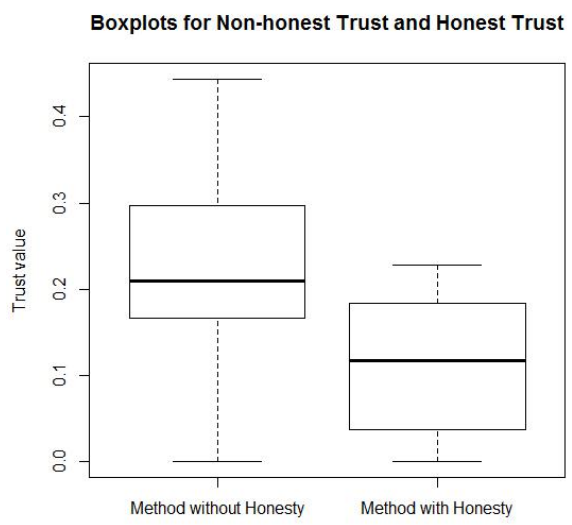

Fig. 3 Boxplots for Trust Data 


\section{Conclusion}

A new trust model based on three factors: familiarity, experience and honesty, was proposed in this paper. These factors which all have degree of uncertainty, are obtained from the interaction that occurs within a community of users. The honesty factor tends to affect the other two factors: familiarity and experience. From the experiments carried out, a better rationale to the inferred trust values were clearly seen from the data with honesty values as compared to that without the honesty value. It was also revealed that honest or accurately assigned values given to individuals based on their importance to roles are likely to be considered for future engagements even though they don't care about the engagement history of other members in the community. Further investigation is required to observe if individuals who were misrepresented or given dishonest values in a community, can influence or motivate other members of the community.

\section{Appendix}

Table 6 Sample data for the experiment

\begin{tabular}{|c|c|c|c|c|c|c|c|c|c|c|c|c|c|c|c|c|c|c|c|c|c|}
\hline $\bar{v}_{1}$ & & & & & & & & & & & & & & & & & & & & & \\
\hline $\mathrm{V}_{2}$ & & 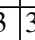 & 30 & 32 & 36 & 44 & 1 & 4 & 26 & 32 & 36 & 44 & 27 & $|90|$ & \begin{tabular}{l|l|l}
7 & 9
\end{tabular} & 19 & 32 & & 32 & & \\
\hline $\mathbf{V}_{3}$ & 13 & 2 & 4 & 1 & 12 & 5 & \begin{tabular}{l|l}
355 & 7 \\
\end{tabular} & 1 & 4 & 19 & 9 & 5 & \begin{tabular}{l|l|}
1 & 1 \\
\end{tabular} & 1 & \begin{tabular}{|l|l}
1 & 6 \\
\end{tabular} & 1 & 8 & & \begin{tabular}{l|l}
6 & 25 \\
\end{tabular} & & \\
\hline $\mid \overline{V_{1}}$ & 19 & 26 & 30 & 32 & 32 & 32 & 32 & 32 & 36 & 36 & $\overline{36}$ & |36 & 36 & 36 & 36 & 4 & 44 & 44 & $\mid 44$ & & 445 \\
\hline $\mathbf{V}_{2}$ & 8 & 7 & 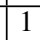 & 1 & 3 & 8 & 9 & 26 & 1 & 3 & 6 & \begin{tabular}{|l|}
19 \\
\end{tabular} & 32 & 44 & 90 & 1 & 3 & 19 & 32 & 36 & 90 \\
\hline $\mathbf{V}_{\mathbf{3}}$ & 4 & 1 & 1 & 1 & 8 & 12 & 6 & 1 & 6 & 5 & 5 & 3 & 2 & 2 & 2 & 4 & 1 & 3 & 2 & 1 & \\
\hline
\end{tabular}

\section{References}

1. Carter, J., Ghorbani, A.A.: Towards a formalization of value-centric trust in agent societies. Web Intelligence \& Agent Systems (1992), pp. 167-183.

2. Erikson, E. H. (1995). Childhood and Society. London: Vintage.

3. Hardin, R. (1985). Trust. Cambridge: Polity Press.

4. Josang, A. and Ismail, R.: The beta reputation system. In Proceedings of the 15th Bled Economic Commerce Conference (2002), Citeseer, pp. 41-55.

5. Luhmann, N.(2000) Familiarity, confidence, trust: Problems and alternatives. In Trust: Making and breaking cooperative relations, Gambetta D.(ed), vol.6 (pp.94-107). University of Oxford, Department of Sociology.

6. Marsh, S. P. (1994). Formalising trust as a computational concept. PhD thesis, University of Stirling. Available at www.cs.stir.ac.uk/research/publications/techreps/pdf/TR133.pdf .

7. Nepal, S., Sherchan, W and Paris, C. Strust: A trust model for social networks. In Proceedings of the 10th International Conference on Trust, Security and Privacy in Computing and Communications (2011), IEEE, pp. 841-846. 
8. Opsahl, T.: Datasets. [Online], n.d. Available at http://toreopsahl.com/datasets/ .

9. R Core Team. R: A language and environment for statistical computing. R Foundation for Statistical Computing, Vienna, Austria, 2014.

10. Rutter, J.: From the sociology of trust towards a sociology of e-trust. International Journal of New Product \& Innovation Management (2001).

11. Söderlund, M. and Gunnarsson, J.: Customer familiarity and its effect on satisfaction and dissatisfaction. SSE/EFI working Paper Series in Business Administration (2000).

12. Stipak, B.: Boxplots. Available at http://web.pdx.edu/ stipakb/download/PA551/boxplot.html, n.d [Accessed 04/08/2015].

13. Wu, J. and Chiclana, F.: A social network analysis trust-consensus based approach to group decision-making problems with interval-valued fuzzy reciprocal preference relations. Knowledge-Based Systems (2014).

14. Zabell, S.: The rule of succession. Erkenntnis (1989), vol 31, pp. 283-321. Available at http://www.researchgate.net/publication/226714676 .

15. Zhang, J., Ghorbani, A. A., Cohen, R.: A familiarity-based trust model for effective selection of sellers in multiagent e-commerce systems. International Journal of Information Security (2007), vol 6, pp. 333-344.

16. Towards real-world models of trust: Reliance on received information. [Online], (1998). Available at http://mcwg.org/mcg-mirror/trustdef.htm . 